\title{
Effects of Boronic Acid on the Fluoride-selective Chemosignaling Behavior of a Merocyanine Dye
}

\author{
Sunyoung Cha, Hye Lim Jeon, Myung Gil Choi, Jong-In Choe, and Suk-Kyu Chang* \\ Department of Chemistry, Chung-Ang University, Seoul 156-756, Korea. *E-mail: skchang@cau.ac.kr \\ Received January 31, 2010, Accepted March 15, 2010
}

\begin{abstract}
The effects of boronic acid on the fluoride-selective chemosignaling behavior of a merocyanine dye were investigated. In the presence of phenylboronic acid (PBA), $N$-methylquinolinium-based merocyanine dye displayed fluoride-selective chromogenic signaling behavior over other commonly coexisting anions in the micromolar concentration range. Signaling is produced by a fluoride-induced displacement of the dye from its complex with PBA, resulting in a significant chromogenic signal for the fluoride ion. This signaling was successfully analyzed using a ratiometric analysis of the UV-vis absorption in response to changes in fluoride ion concentration. A PBA substituted with an electron withdrawing group was found to exhibit a more pronounced signal. Polymer-bound PBA also exhibited useful fluoride-selective signaling behavior.
\end{abstract}

Key Words: Fluoride, Chemosignaling, Merocyanine dye, Phenylboronic acid, Ratiometry

\section{Introduction}

Anionic species play important roles in many chemical and biological processes and a number of optical chemosensors for the selective detection of anionic substrates of different shapes and charges have been reported. ${ }^{1,2}$ Of the many important anionic targets, the fluoride ion has attracted much research interest due to its importance in many chemical, biological, clinical, and environmental science processes. ${ }^{3}$ For example, highly selective fluoride sensors are useful for the determination of fluoride levels in municipal/potable water and for monitoring organofluorophosphate substances commonly used as chemical warfare agents.

There are a variety of sophisticated fluoride sensors that have been developed. ${ }^{5}$ Representative sensors operating in organic solvents are made up of various compounds based on unmodified fluorescein, ${ }^{6}$ bisurea of anthracene, ${ }^{7}$ pyrrolylquinoxalines containing pyrene antenna moieties, ${ }^{8}$ and calix[4]arene bearing naphthocrown and coumarin units. ${ }^{9}$ In addition, chemodosimeters for fluoride anions based upon well-known boron-dipyrromethene dyes ${ }^{10}$ and axial-substituted subphthalocyanine moiety ${ }^{11}$ have also been successfully devised. The selective deprotection of silyl ether groups as a handle for fluoride signaling has also attracted much research interest, and resorufin, ${ }^{12}$ fluorescein, ${ }^{13}$ as well as coumarin ${ }^{14}$ derivatives have been successfully developed. Another interesting system reported by Kim and Swager is a turn-on type dosimeter utilizing fluoride-triggered coumarin dye formation via the unique chemical reactivity of fluoride with bulky silyl-protecting groups. ${ }^{15}$

In parallel to these, various functionalized boron compounds have been widely used as signaling systems due to the high affinity of fluoride for boron atoms. Chelating and cationic boranes which form highly stable fluoroborates ${ }^{16}$ and boron-containing $\pi$-conjugated systems including tri (9-anthryl) borane and tris[(10-dimesitylboryl)-9-anthryl]borane ${ }^{17}$ are typical systems that have been used as fluoride sensors. A fluorescein derivative bearing a boronic acid, ${ }^{18}$ Lewis acid-base interaction system between a trivalent boron atom and fluoride anion, ${ }^{19}$ and boryl-functionalized dithienophosphole oxide ${ }^{20}$ have also been reported for this purpose.

Recently, interesting fluoride signaling systems based on the fluoride-induced displacement of a dye from the complex formed between Brooker's merocyanine dye and phenylboronic acid (PBA) or calix[4]pyrrole have been reported. ${ }^{21}$ In this paper, we report a chemosignaling system based on a similar structured merocyanine dye 1 that contains a quinolinium moiety and PBA. In particular, a series of PBAs with different substituents were employed to investigate substituent effects on the fluoride signaling behavior of the 1-PBA system. Polymeric PBA was also tested as a fluoride-selective signaling system with the aims of convenience in measurements and of reagent reusability.

\section{Results and Discussion}

Compound 1 was prepared by the reaction of 4-formylphenol with $N$-methyllepidinium iodide, ${ }^{22}$ which was available from the $\mathrm{N}$-methylation of lepidine with $\mathrm{CH}_{3} \mathrm{I}$, in the presence of piperidine in ethanol. The initially prepared compound was treated with a strongly basic anionic exchange resin (Amberlite IRA410 ) to ensure the betaine type structure of merocyanine 1.

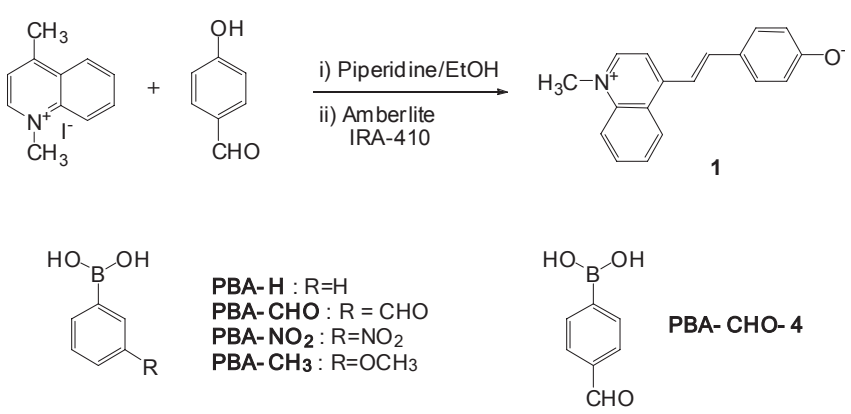

Scheme 1. Synthesis of merocyanine dye and structures of phenylboronic acid derivatives used for the fluoride-selective signaling system 


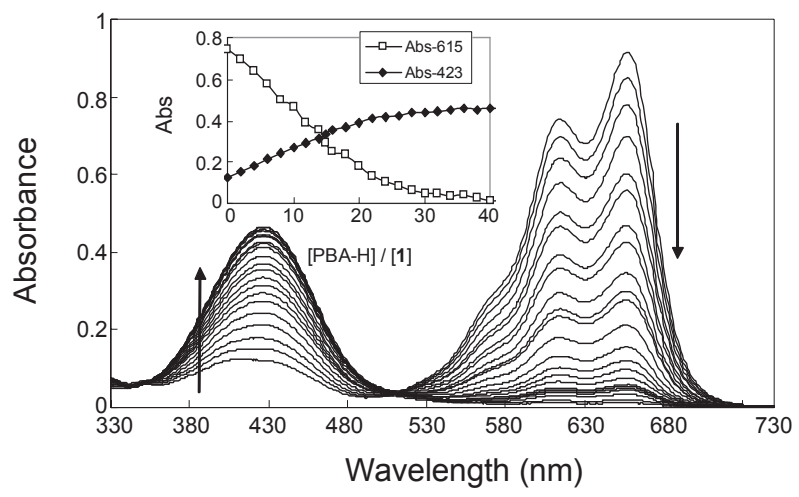

Figure 1. Changes in the UV-vis absorption spectra of 1 in the presence of varying amounts of PBA-H in acetonitrile. $[1]=2.0 \times 10^{-5} \mathrm{M}$.

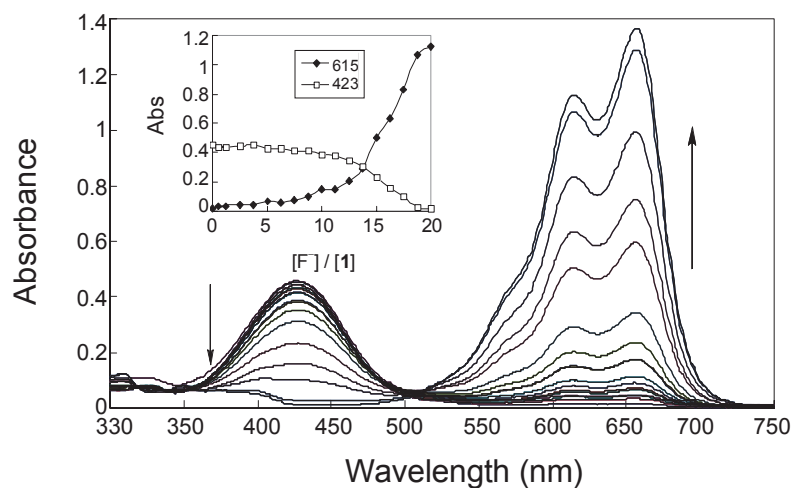

Figure 2. Changes in the UV-vis absorption spectra of 1-PBA-H as a function of fluoride ion concentration in acetonitrile. $[1]=2.0 \times 10^{-5} \mathrm{M}$, $[\mathrm{PBA}-\mathrm{H}]=1.0 \times 10^{-3} \mathrm{M}$.

The absorption behavior of the dye 1 upon interaction with phenylboronic acid (PBA-H) was initially investigated. Dye $\mathbf{1}$ showed strong absorption bands at 615 and $655 \mathrm{~nm}$ and a weak band around $423 \mathrm{~nm}$ in acetonitrile. Upon treatment with PBA$\mathrm{H}$, the absorption bands at 615 and $655 \mathrm{~nm}$ steadily decreased while the weak band at $423 \mathrm{~nm}$ increased (Figure 1). With 40 equiv of PBA-H, the absorption bands at 615 and $655 \mathrm{~nm}$ almost completely disappeared, with a concomitant change in solution color from blue to yellow.

Next, the selective signaling behavior of the 1-PBA-H system toward representative anions was investigated. The yellow solution of 1-PBA-H $\left([1]=2.0 \times 10^{-5} \mathrm{M}\right.$ and $\left.[\mathrm{PBA}-\mathrm{H}]=1.0 \times 10^{-3} \mathrm{M}\right)$ was treated with various anions, and the absorptions of the resulting solutions were measured. With an increasing amount of fluoride ion, the absorbance of the 1-PBA-H system at $423 \mathrm{~nm}$ decreased while the bands at 615 and $655 \mathrm{~nm}$ steadily increased (Figure 2). The yellow solution of 1-PBA-H progressively changed to a blue solution. With 20 equiv of fluoride ion, the solution color was almost identical to that of $\mathbf{1}$ alone. Other representative anions exhibited almost no change in absorption spectra, except for the acetate ion which exhibited less pronounced increases in absorption at 615 and $655 \mathrm{~nm}$ compared with fluoride ion (inset of Figure 3). The selectivity toward the fluoride ion could be more easily visualized by ratiometry using the absorbances at 615 and $423 \mathrm{~nm}$. The absorbance ratio $\left(A_{615} /\right.$

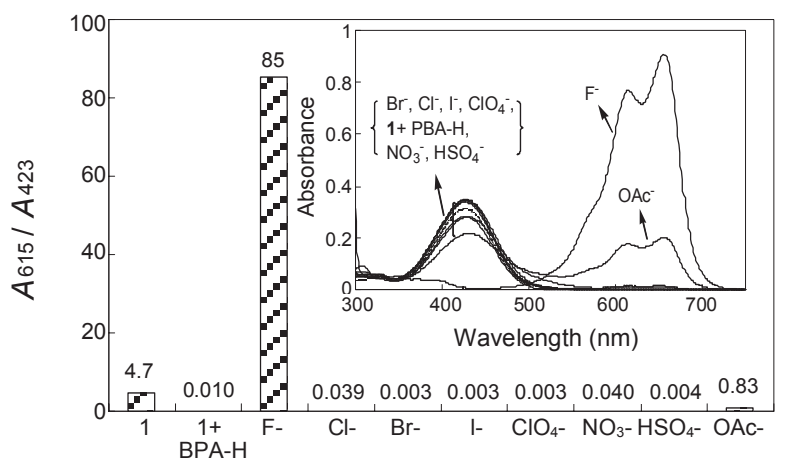

Figure 3. Absorbance ratios $\left(A_{615} / A_{423}\right)$ of 1-PBA-H in the presence of various anions in acetonitrile. $[1]=2.0 \times 10^{-5} \mathrm{M},[\mathrm{PBA}-\mathrm{H}]=1.0 \times 10^{-3}$ $\mathrm{M},[$ Anion $]=4.0 \times 10^{-4} \mathrm{M}$.
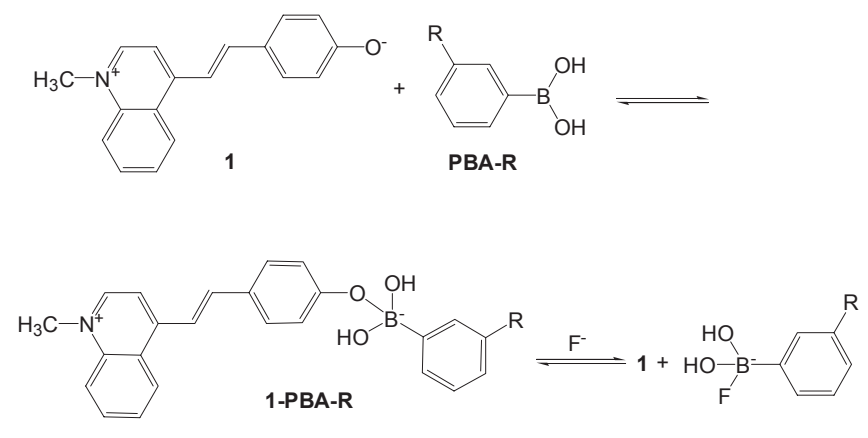

Scheme 2. Mechanism of fluoride-selective signaling by 1-PBA.

$\left.A_{423}\right)$ of the 1-PBA-H system in the absence of any anion was 0.01 ; however, with the introduction of fluoride, the ratio was greatly enhanced to 85.3 , while the acetate ion produced only a moderate increase to 0.83 (Figure 3 ). Other tested anions showed minimal changes, ranging from 0.003 for bromide to 0.04 for nitrate.

As depicted in Scheme 2, fluoride-selective signaling is due to the interaction of the merocyanine dye 1-PBA complex with a fluoride ion, which in a selective displacement yields the free dye $\mathbf{1}$. The displaced dye 1 exhibited a characteristic absorption that is dependent on the concentration of fluoride ions.

In order to gain a better understanding of the signaling behavior of the 1-PBA system, we investigated effects of substituents on the PBA on fluoride signaling. The interactions between 1 and the PBA derivatives were found to be strongly affected by the nature of the substituent and could be easily monitored using the ratio of the two absorbances at 615 and 423 $\mathrm{nm}$ (Figure 4). As the electron withdrawing effect of the substituents increased, the complex formation between 1 and PBA became more effective. For example, in the titration of 1 with $\mathrm{PBA}-\mathrm{CHO}$ or $\mathrm{PBA}-\mathrm{NO}_{2}$, larger changes in the absorbances were observed compared with that of PBA-H. In fact, the ratio $A_{615} /$ $A_{423}$ of 1-PBA-CHO and 1-PBA-NO ${ }_{2}$ reached baseline with about 5 equiv of $\mathrm{PBA}$, which is much smaller than the 40 equiv of PBA-H needed for a similar effect. Electronic effects on the 


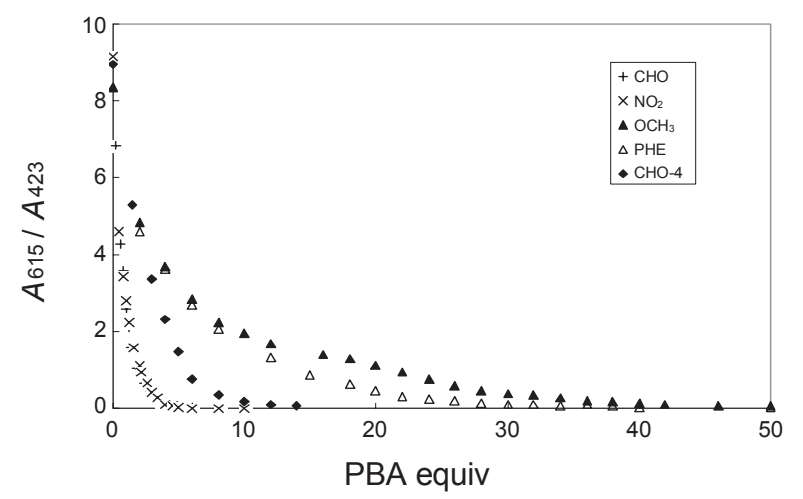

Figure 4. UV-vis titration of $\mathbf{1}$ with various PBA derivatives in acetonitrile. $[\mathbf{1}]=2.0 \times 10^{-5} \mathrm{M}$.

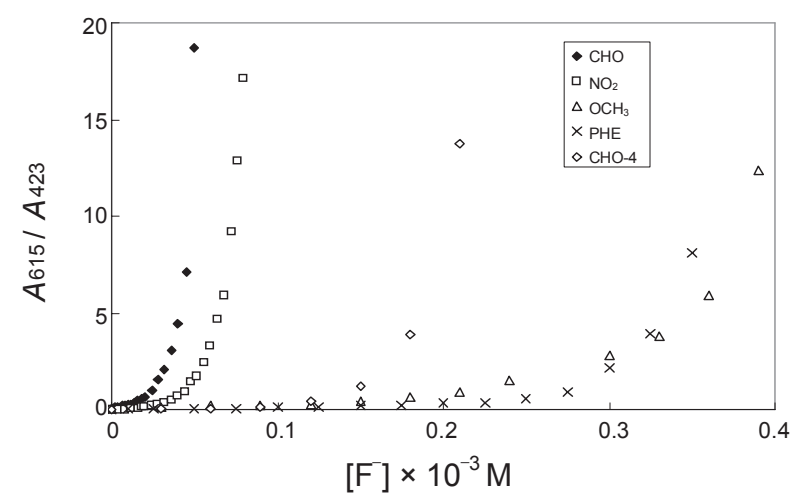

Figure 5. UV-vis titration of the 1-PBA system with fluoride ions in acetonitrile. $[1]=2.0 \times 10^{-5} \mathrm{M}$ and $[\mathrm{PBA}]=1.0 \times 10^{-4} \mathrm{M}$ for PBA-CHO and PBA-NO $2,3.0 \times 10^{-4} \mathrm{M}$ for PBA-CHO- $4,1.0 \times 10^{-3} \mathrm{M}$ for PBA-H and PBA-OCH ${ }_{3}$.

interaction were further supported by the observation that the absorbance changes with $\mathrm{PBA}-\mathrm{OCH}_{3}$ were somewhat less efficient than were those with PBA-H, as the decrease in $A_{615} / A_{423}$ with 1-PBA-OCH${ }_{3}$ reached baseline with about 50 equiv of PBA$\mathrm{OCH}_{3}$. To check the possible effects of positional isomer, 4-formylphenylboronic acid (PBA-CHO-4) was tested. The interaction of PBA-CHO-4 with 1 was found to be less effective than 3 -formyl isomer and the strength of the 1-PBA complexes decreased in the following sequence: $\mathrm{PBA}-\mathrm{CHO}>\mathrm{PBA}-\mathrm{NO}_{2}>$ PBA-CHO- $4>$ PBA-H $>$ PBA-OCH ${ }_{3}$. These trends were further supported by the association constant measurements. Nonlinear curve fitting $^{23}$ of the absorbance changes of $\mathbf{1}$ as a function of PBA at longer wavelength around $654 \mathrm{~nm}$ afforded $\mathrm{K}_{\mathrm{a}}$ values for 1-PBA complexes of $5.1 \times 10^{4}, 2.4 \times 10^{4}, 8.3 \times 10^{3}, 1.9 \times 10^{3}$, and $1.1 \times 10^{3} \mathrm{M}^{-1}$ for PBA-CHO, PBA-NO $\mathrm{N}_{2}$ PBA-CHO-4, $\mathrm{PBA}-\mathrm{H}$, and $\mathrm{PBA}-\mathrm{OCH}_{3}$, respectively.

For insight into the quantitative analytical behavior of the 1-PBA system, a UV-vis titration of the 1-PBA system with fluoride in acetonitrile was performed. The fluoride ion induced a more pronounced signaling with 1-PBA-CHO than with 1-PBAH. As shown in Figure 5, the slope of the titration curve with 1-PBA-CHO was much steeper than that with 1-PBA-H, which suggests that the 1-PBA-CHO system, with an electron withdrawing substituent, is more effective in fluoride signaling under

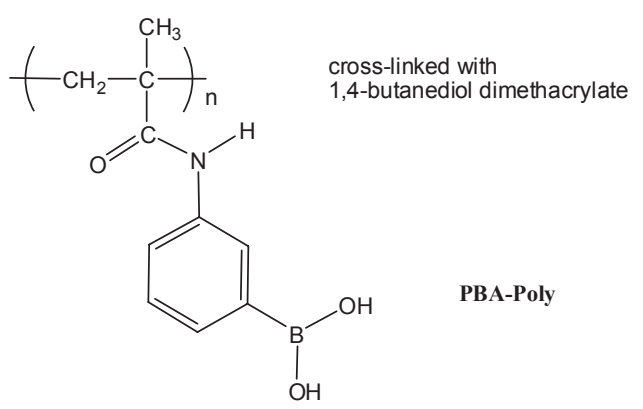

Figure 6. Structure of polymer-bound phenylboronic acid, PBA-Poly.

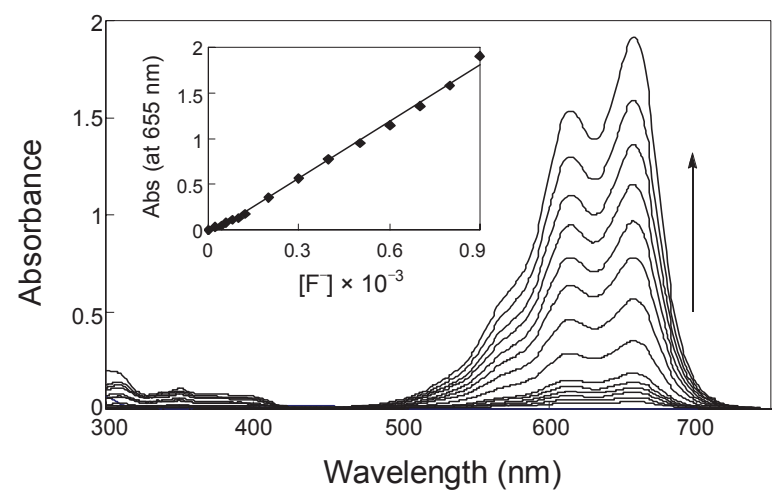

Figure 7. UV-vis titration of polymer-bound phenylboronic acid, PBAPoly, with fluoride ions. Dye absorbed PBA-Poly $=2.0 \mathrm{mg}$ in $3.0 \mathrm{~mL}$ acetonitrile.

the employed conditions. The effectiveness of the signaling decreased in the sequence of $\mathrm{PBA}-\mathrm{CHO}>\mathrm{PBA}_{-} \mathrm{NO}_{2}>\mathrm{PBA}-$ $\mathrm{CHO}-4>\mathrm{PBA}-\mathrm{H}>\mathrm{PBA}-\mathrm{OCH}_{3}$. From the changes in the fluoride-dependent absorbance ratio, $A_{615} / A_{423}$, the detection limit $^{24}$ of the most sensitive 1-PBA-CHO system for measuring fluoride was estimated to be $6.1 \times 10^{-6} \mathrm{M}$ in acetonitrile.

Fluoride signaling of the 1-PBA system was relatively fast, reaching completion within 2 min of sample preparation. Other possible interfering anions, such as chloride, bromide, iodide, hydrogenphosphate, and hydrogensulfate, did not produce any significant responses up to $24 \mathrm{~h}$ after sample preparation. Furthermore, the mildly interfering acetate ion did not exhibit any significant changes in $A_{615} / A_{423} 24 \mathrm{~h}$ after sample preparation. The possibility of signaling in aqueous solution was also surveyed by checking the effects of water on the formation of the 1-PBA system in acetonitrile. As the water content was increased, formation of the 1-PBA complex decreased, and the 1-PBA structure was maintained only up to a content of $4 \%$ water, as was evident in the UV-vis measurements. Furthermore, the fluoride-selective signaling behavior was less pronounced in $4 \%$ aqueous acetonitrile than it was in $100 \%$ acetonitrile. $^{25}$

Finally, application of the 1-PBA system in the signaling of fluoride ions was attempted using a commercially available boronic acid functionalized polymer. Polymer-bound 3-amido- 
phenylboronic acid, PBA-Poly, which is a cross-linked copolymer of dihydroxyborylanilino-substituted methacrylic acid with 1,4-butanediol dimethacrylate (boron content $=1.4 \%$ (dry), 0.1 $0.4 \mathrm{~mm}$ bead size), was used as a signaling component (Figure 6). Upon treatment of PBA-Poly with dye 1, a deep orange-colored bead was obtained. The dye-absorbed polymer was similarly titrated with fluoride ion, and the UV-vis absorptions were measured in acetonitrile. As can be seen from Figure 7, the changes in absorbance of the solution reveal a well-defined titration curve in response to changes in fluoride ion concentration quite similar to that of the small molecule 1-PBA system. Selective signaling was possible in the range between $2.0 \times 10^{-5}$ and $1.0 \times 10^{-3} \mathrm{M}$, and the detection limit was $9 \times 10^{-6} \mathrm{M}$. The observed concentration-dependent absorbance changes of PBA-Poly suggest that the dye-absorbed polymer could be used as a selective signaling system for fluoride ions. Furthermore, due to the covalent nature of the boronic acid, the polymer system could be used repetitively after a simple treatment with the dye solution.

In summary, a simple fluoride-selective chemosignaling system based on an easily available merocyanine dye and phenylboronic acid was investigated. The fluoride-induced dissociation of the dye complex into individual components resulted in significant chromogenic signaling in acetonitrile solution. The signaling efficiency was strongly dependent on the substituents of the phenylboronic acid, and aldehyde and nitro derivatives were found to be the most suitable for pronounced fluorideselective signaling. Polymer-bound phenylboronic acid also exhibited useful signaling behavior.

\section{Experimental Section}

General. Phenylboronic acid, 3-formylphenylboronic acid, 3-nitrophenylboronic acid, 3-methoxyphenylboronic acid, 4methylquinoline, 4-hydroxybenzaldehyde, and phenyl boronic acid gel were purchased from Aldrich Chemical Co. and were used without further purification. UV-vis spectra were collected with a Jasco V-550 spectrophotometer. 'Anhydrous grade' acetonitrile, used for the measurements of UV-vis spectra, was obtained from Aldrich Chemical Co. ${ }^{1} \mathrm{H} \mathrm{NMR}(300 \mathrm{MHz})$ and ${ }^{13} \mathrm{C}$ NMR (75 MHz) were obtained on a Varian Gemini 2000 spectrometer. Chemical shifts were reported relative to the residual peaks of DMSO. Mass spectral data were obtained with a Micromass Autospec mass spectrometer.

Synthesis of $\mathbf{N}$-methyllepidinium iodide. ${ }^{22}$ To a solution of lepidine $(500 \mathrm{mg}, 3.5 \mathrm{mmol})$ in dichloromethane was added methyl iodide (1.5 $\mathrm{mL}, 25 \mathrm{mmol})$, and the mixture was stirred at room temperature for one day. The precipitated solid was filtered and dried under air to yield the yellow colored $N$-methyllepidinium iodide. Yield 98\%. ${ }^{1} \mathrm{H}$ NMR (300 MHz, DMSO-d 6 ) 9.35 $(\mathrm{d}, J=6.0 \mathrm{~Hz}, 1 \mathrm{H}), 8.55-8.47(\mathrm{~m}, 2 \mathrm{H}), 8.30-8.24(\mathrm{~m}, 1 \mathrm{H}), 8.10-$ $8.04(\mathrm{~m}, 2 \mathrm{H}), 4.58$ (s, 3H), 3.00 (s, 3H).

Synthesis of 1. To a solution of $N$-methyllepidinium iodide (143 mg, $0.5 \mathrm{mmol}$ ) in ethanol was added 4-formylphenol (73 $\mathrm{mg}, 0.6 \mathrm{mmol}$ ) and piperidine $(85 \mathrm{mg}, 1 \mathrm{mmol})$, and the solution was refluxed for $1 \mathrm{~h}$. After cooling in an ice-water bath, precipitated solid was filtered and washed with ethanol and water. The resulting dark-colored powder was dried under vacuum.
The product was finally treated with an anion exchange resin (Amberlite IRA-410) in acetonitrile to obtain betaine structure of 1. Yield 86.6\%. ${ }^{1} \mathrm{H}$ NMR (300 MHz, DMSO- $\left.d_{6}\right) 9.25$ (d, $J=$ $6.6 \mathrm{~Hz}, 1 \mathrm{H}), 9.04(\mathrm{~d}, J=8.3 \mathrm{~Hz}, 1 \mathrm{H}), 8.43(\mathrm{~d}, J=6.6 \mathrm{~Hz}, 1 \mathrm{H})$, $8.41(\mathrm{~d}, J=8.7 \mathrm{~Hz}, 1 \mathrm{H}), 8.27-8.23(\mathrm{~m}, 1 \mathrm{H}), 8.14(\mathrm{~s}, 2 \mathrm{H}), 8.05-$ $8.00(\mathrm{~m}, 1 \mathrm{H}), 7.88(\mathrm{~d}, J=8.4 \mathrm{~Hz}, 2 \mathrm{H}), 6.91(\mathrm{~d}, J=8.4 \mathrm{~Hz}, 2 \mathrm{H})$, $4.51(\mathrm{~s}, 3 \mathrm{H}) .{ }^{13} \mathrm{C}$ NMR $\left(150 \mathrm{MHz}, \mathrm{DMSO}-d_{6}\right) 161.0,158.5$, 148.0, 144.0, 139.2, 135.3, 131.7, 129.4, 127.2, 126.9, 126.5, $119.7,116.5,116.4,115.7,44.9$. HRMS (EI); $\mathrm{m} / \mathrm{z}$ calcd for $\mathrm{C}_{18} \mathrm{H}_{16} \mathrm{NO}[\mathrm{M}]^{+}:$262.1232, found 262.1256.

Fluoride signaling. An acetonitrile solution of dye $1(20 \mu \mathrm{M})$ was titrated with phenylboronic acid just until the solution color changed from blue to yellow. To this solution were added incremental amounts of tetrabutylammonium fluoride (in acetonitrile), and the UV-vis absorption spectra were measured. From these titration results, the detection limit was estimated by plotting the changes in absorbance with $\log \left[\mathrm{F}^{-}\right]$. The detection limit was calculated from the intersection of the linear part of the plot with the $\mathrm{x}$-axis following a reported procedure. ${ }^{23}$

Similarly, polymer-bound phenylboronic acid, PBA-Poly, was tested as a signaling system for fluoride ions. PBA-Poly (2.0 $\mathrm{mg}$ ) was treated with an excess of dye solution (200 mM) until the solution exhibited the blue color of excess dye 1 . After $1 \mathrm{~h}$, the deep orange-colored polymer bead was separated and washed with acetonitrile to yield the dye-absorbed polymer. The dyeabsorbed polymer in acetonitrile was treated with incremental amounts of tetrabutylammonium fluoride $(10 \mathrm{mM}$ in acetonitrile), and the UV-vis absorption spectra were measured.

Acknowledgments. This work was supported by National Research Foundation of Korea Grant funded by the Korean Government (No. 2009-0072415).

\section{References}

1. Binachi, A.; Bowman-James, K.; Garcia-Espana, E. Supramolecular Chemistry for Anions; John Wiley \& Sons: New York, 1997. (b) Stibor, I. Anion Sensing, Topics in Current Chemistry 255; Springer-Verlag: New York, 2005. (c) Sessler, J. L.; Davis, J. M. Acc. Chem. Res. 2001, 34, 989.

2. Martínez-Máñez, R.; Sancenón, F. Chem. Rev. 2003, 103, 4419. (b) Gunnlaugsson, T.; Glynn, M.; Tocci, G. M.; Kruger, P. E.; Pfeffer, F. M. Coord. Chem. Rev. 2006, 250, 3094.

3. Ayoob, S.; Gupta, A. K. Crit. Rev. Environ. Sci. Technol. 2006, 36, 433. (b) Bassin, E. B.; Wypij, D.; Davis, R. B. Cancer Cause Control 2006, 17, 421. (c) Yu, Y.; Yang, W.; Dong, Z.; Wan, C.; Zhang, J.; Liu, J.; Xiao, K.; Huang, Y.; Lu, B. Fluoride 2008, 41, 134.

4. Badr, I. H. A.; Meyerhoff, M. E. Anal. Chem. 2005, 77, 6719.

5. (a) Cho, E. J.; Moon, J. W.; Ko, S. W.; Lee, J. Y.; Kim, S. K.; Yoon, J.; Nam, K. C. J. Am. Chem. Soc. 2003, 125, 12376. (b) Xu, Z.; Kim, S. K.; Han, S. J.; Lee, C.; Kociok-Kohn, G.; James, T. D.; Yoon, J. Eur. J. Org. Chem. 2009, 3058. (c) Yeo, H. M.; Ryu, B. J.; Nam, K. C. Org. Lett. 2008, 10, 2931. (d) Cametti, M.; Rissanen, K. Chem. Commun. 2009, 2809.

6. Zhang, X.; Shiraishi, Y.; Hirai, T. Tetrahedron Lett. 2007, 48, 8803.

7. Kim, S. K.; Yoon, J. Chem. Commun. 2002, 770.

8. Pohl, R.; Aldakov, D.; Kubát, P.; Jursíková, K.; Marquez, M.; Anzenbacher, P., Jr. Chem. Commun. 2004, 1282.

9. Lee, M. H.; Quang, D. T.; Jung, H. S.; Yoon, J.; Lee, C.-H.; Kim, J. S. J. Org. Chem. 2007, 72, 4242.

10. Coskun, A.; Akkaya, E. U. Tetrahedron Lett. 2004, 45, 4947.

11. Xu, S.; Chen, K.; Tian, H. J. Mater. Chem. 2005, 15, 2676. 
12. (a) Kim, S. Y.; Hong, J.-I. Org. Lett. 2007, 9, 3109. (b) Kim, S. Y.; Park, J.; Koh, M.; Park, S. B.; Hong, J.-I. Chem. Commun. 2009, 4735.

13. Yang, X.-F.; Ye, S.-J.; Bai, Q.; Wang, X.-Q.J. Fluoresc. 2007, 17, 81.

14. Yang, X.-F. Spectrochim. Acta A 2007, 67, 321.

15. Kim, T.-H.; Swager, T. M. Angew. Chem. Int. Ed. 2003, $42,4803$.

16. (a) Hudnall, T. W.; Chiu, C.-W.; Gabbaï, F. P. Acc. Chem. Res. 2009, 42, 388. (b) Kim, Y.; Gabbaï, F. P. J. Am. Chem. Soc. 2009, 131,3363 .

17. Yamaguchi, S.; Akiyama, S.; Tamao, K. J. Am. Chem. Soc. 2001, $123,11372$.

18. Swamy, K. M. K.; Lee, Y. J.; Lee, H. N.; Chun, J.; Kim, Y.; Kim, S.-J.; Yoon, J. J. Org. Chem. 2006, 71, 8626.

19. Liu, Z.-Q.; Shi, M.; Li, F.-Y.; Fang, Q.; Chen, Z.-H.; Yi, T.; Huang,
C.-H. Org. Lett. 2005, 7, 5481.

20. Neumann, T.; Dienes, Y.; Baumgartner, T. Org. Lett. 2006, 8, 495.

21. (a) Nicolini, J.; Testoni, F. M.; Schuhmacher, S. M.; Machado, V. G. Tetrahedron Lett. 2007, 48, 3467. (b) Linn, M. M.; Poncio, D. C.; Machado, V. G. Tetrahedron Lett. 2007, 48, 4547.

22. Coe, B. J.; Foxon, S. P.; Harper, E. C.; Harris, J. A.; Helliwell, M.; Raftery, J.; Asselberghs, I.; Clays, K.; Franz, E.; Brunschwig, B. S.; Fitch, A. G. Dyes Pigm. 2009, 82, 171.

23. Kuzmič, P. Anal. Biochem. 1996, 237, 260.

24. Shortreed, M.; Kopelman, R.; Kuhn, M.; Hoyland, B. Anal. Chem. 1996, 68, 1414.

25. The possibility of using fluorescence measurements for fluoride signaling with the present system was also studied; however, the fluorescence changes of 1-PBA system upon interactions with various anions were not as pronounced as the UV-vis chromogenic signaling behavior. 